\title{
Cellular IOT using nRF9160kit
}

\author{
https://doi.org/10.3991/ijoe.v16i15.18987 \\ Arvind Vishnubhatla \\ Gokaraju Rangaraju institute of engineering and Technology, Hyderabad, India \\ vainfo66@gmail.com
}

\begin{abstract}
The current vision of internet of things aims at connecting anything with everything. It is estimated that there will be 18 billion connected devices in 2022. Applications like utility meters, robotics, smart street lighting, process automation, solar and wind farms are expected to grow. High end requirements for automated driving, industrial automation and e-health exist. Cellular IOT is expected to bring new use cases to address latest requirements in the market. There is a need to provide large coverage in a power efficient manner while providing a high battery life. There is a need to have a kit which connects seamlessly and has a small form factor. The requirements on latency and throughput are relaxed in some cases while stringent in others. Stringent requirements make use of more radio resources. There is increased demand for system capacity and network availability. In this paper we make use of nRF9160 kit a low-cost device where a reduction in the cost and complexity has been achieved. The performance objectives of coverage, throughput, latency, capacity, power efficiency and complexity are met. This kit provides a reliable and future proof solution in the long term. The kit is built for the global market and allows roaming over multiple networks.
\end{abstract}

Keywords - Cellular IOT, coverage, latency, throughput, system capacity, network availability, MQTT, cloud

\section{Introduction}

\subsection{Background}

The current vision of internet of things aims at connecting anything with everything. It is estimated that there will be 18 billion connected devices in 2022[1]. Applications like utility meters, robotics, smart street lighting, process automation, solar and wind farms are expected to grow. High end requirements for automated driving, industrial automation and e-health exist. Cellular IOT is expected to bring new use cases to address latest requirements in the market [2]. There is a need to provide large coverage in a power efficient manner while providing a high battery life. There is a need to have a kit which connects seamlessly and has a small form factor [3]. The requirements on latency and throughput are relaxed in some cases while stringent in others. Stringent requirements make use of more radio resources. There is increased demand for system capacity and network availability [4]. In this paper we make use of an nRF9160 kit a 
low-cost device where a reduction in the cost and complexity has been achieved. The performance objectives of coverage, throughput, latency, capacity, power efficiency and complexity are met [5]. This kit provides a reliable and future proof solution in the long term. The kit is built for the global market and allows roaming over multiple networks.

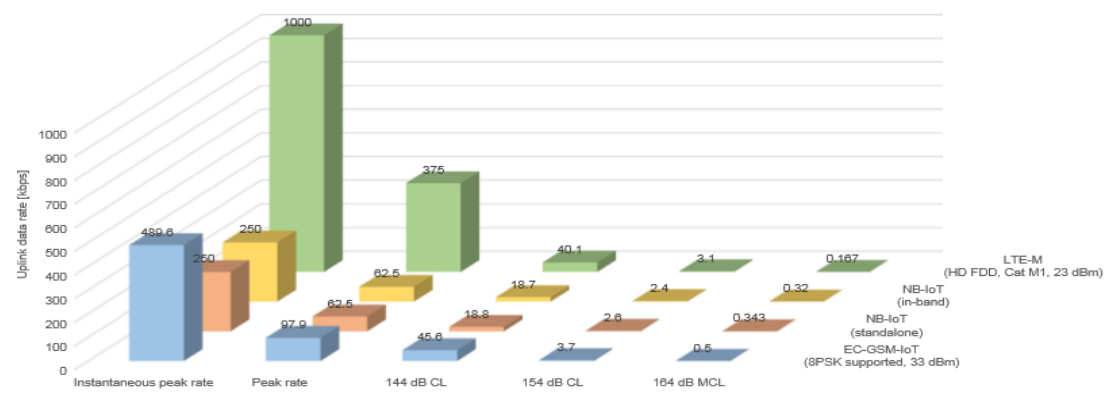

Fig. 1. Coverage and physical layer data rate for uplink

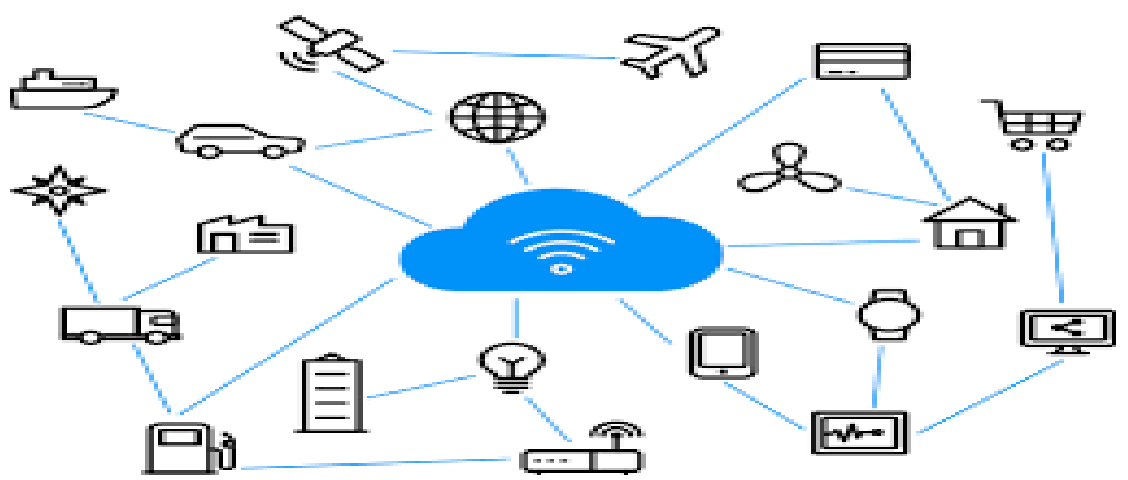

Fig. 2. Applications of IOT

The major need for Cellular IoT in healthcare comes from in-patient care, predictive and preventative health care, hospital equipment tracking and elderly tracking.

Some needs in agriculture include smart irrigation, smart lighting, livestock health, preventative maintenance of farm equipment, robotic farming.

There is need for Cellular IoT in pollution control, weather prediction, waste management, smart traffic light control, city lighting, smart cameras to prevent crime, smart parking, monitoring bridge and street infrastructure.

Other areas where thousands of sensors are needed are oil rig analysis, solar panel monitoring, hazardous nuclear facilities, blade adjustments of remote wind turbines and smart electric meters [6].

Low power wide area networks typically use internet transport protocols and constrained devices like MQTT [7]. The information propagates to the cloud in a large quantity. It is necessary to deal with volume data and extract complex event processing 
using data analytics and machine learning techniques [8]. In addition, each level starting from sensors, CPU, radio communication systems and communication protocols need to incorporate security. There is also the need for authenticity and integrity of the data collected. IOT provides the maximum potential for attackers to penetrate systems and manipulate systems [9].

Cyber Security is an important ingredient of the development life cycle. It is important for digital devices to stay running, as any disruption can tantamount to high economic loss [10].

There is also a need to address the operation of a large number of legacy devices. It should be possible to move seamlessly legacy systems with several thousands of sensors and controllers to the new paradigm. A large number of legacy topologies must meet the standards of robustness, resilience and availability.

\section{Project Methodology}

As very few vendors exist in the market who have tested readymade solutions in the market the quest for easy and reliable Cellular IoT is difficult. One of the first vendors who have demonstrated the capability of Cellular IoT is Nordic Semiconductor with the nRF9160 kit. It is in order for us to explore this solution to demonstrate our capability to address the various Cellular IoT needs.

Shown below is an nRF9160 DK board from Nordic semiconductor for Cellular IoT. The kit has a dedicated application processor and memory. An LTE-M/NB-IoT model modem has been incorporated and GPS support have been incorporated using low power consumption. It provides $700-2200 \mathrm{MHz}$ LTE band support with a $23 \mathrm{dBm}$ output power. A $64 \mathrm{MHz}$ Arm ${ }^{\circledR}$ Cortex ${ }^{\circledR}-\mathrm{M} 33 \mathrm{CPU}$ has been incorporated where the application code can be written. It provides $1 \mathrm{MB}$ Flash \& 256KB RAM. The kit provides automated power and clock management. There is a provision to have 32 GPIOs.
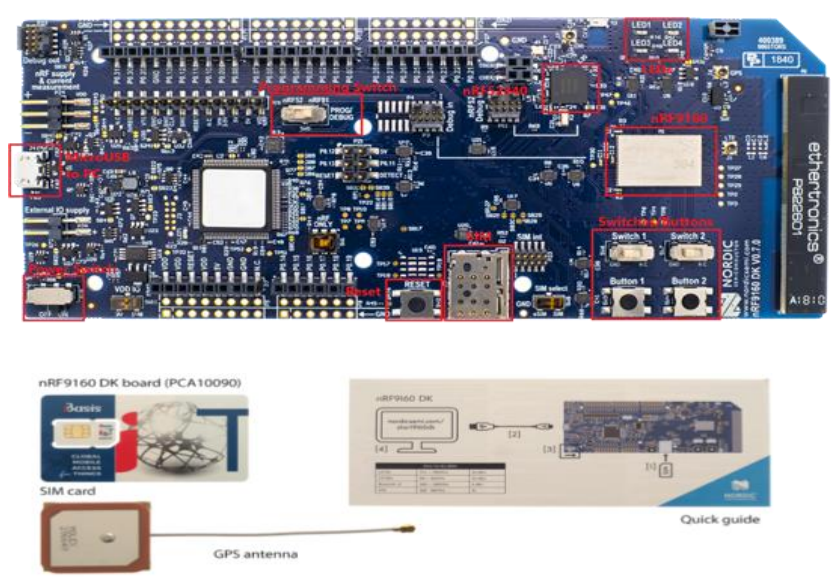

Fig. 3. nRF9160DK board 


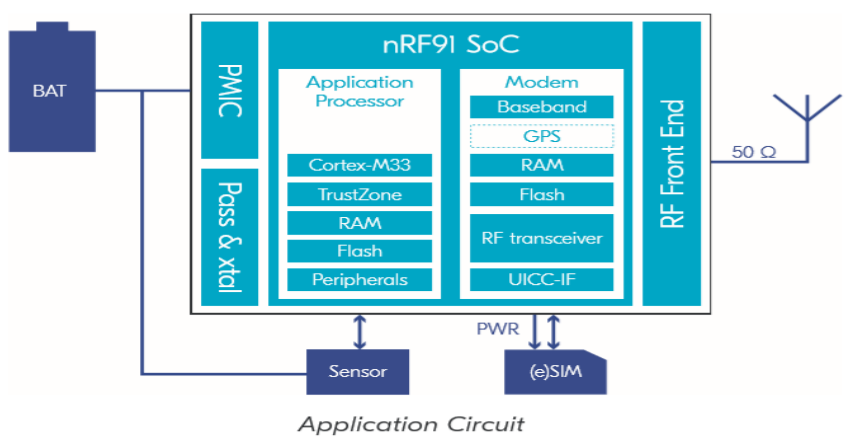

Fig. 4. Application Circuit

Figure 4 shows the conceptual representation of an application processor and an LTE modem. The sensors are interfaced to the applications processor. The SOC connects to the RF front end through an antenna. The bootloader boots the chip and runs application tasks which in turn interface with the LTE Modem. This illustrated in Figure 5.

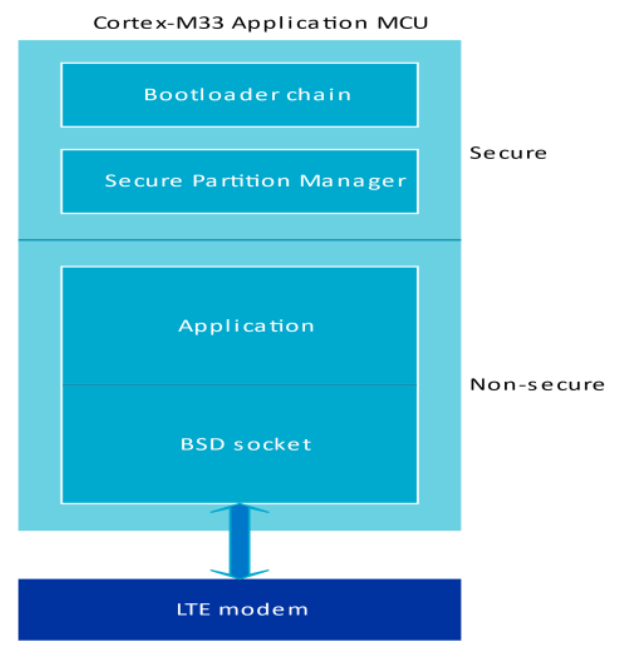

Fig. 5. Conceptual Partition

The code for the application is written in $\mathrm{C}$ language and is compiled using a Cmake utility. Figure 6 shows a tree structure to make the project with a project configuration file and a simple main program.

The nRF connect SDK helps you jump start your application. It makes use of Zephyr RTOS and communicates with CoAP, MQTT and LWM2M. This is an affordable single board development kit for nRF9160SP.It supports both SIM and ESIM. 


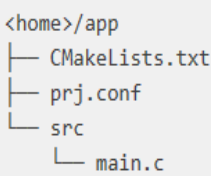

Fig. 6. Application tree

A range of analog and digital peripherals along with cryptographic and security features is built to address internet security and authentication. The LTE modem integrates RFFE radio and baseband. Figures 7 and 8 provide a detailed architectural representation of the board.

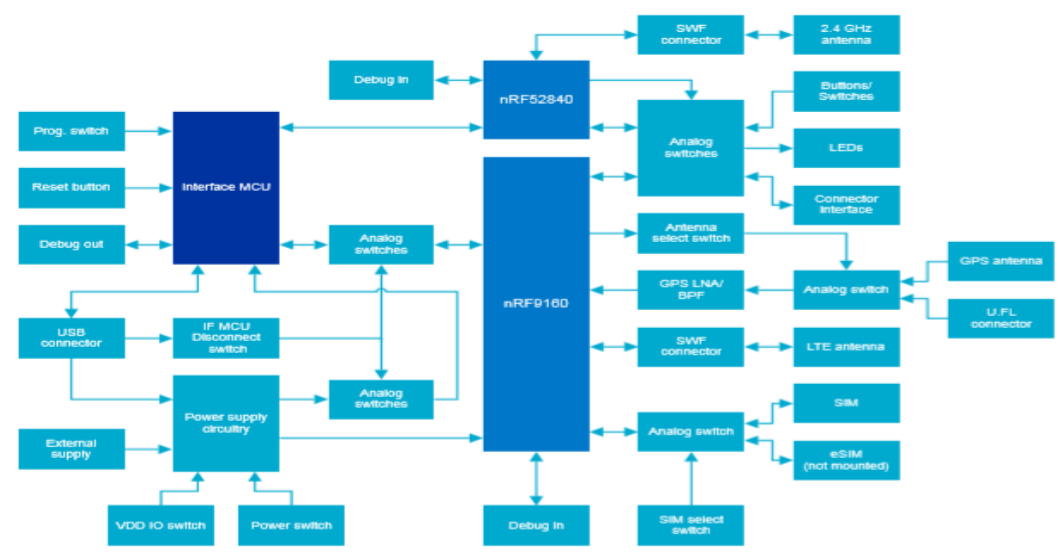

Fig. 7. Architecture diagram of the board

Figure 7 shows that in addition to nRF9160 another processor nRF52840 is also present on the kit.

The nRF52840 is a multi-protocol system on chip with highly flexible capabilities for short range wireless applications. It has an embedded $2.4 \mathrm{GHz}$ transceiver. It supports concurrent Bluetooth 5 and thread wireless connectivity. It is built around a 32bit 64MHz Arm Cortex-M4F CPU with 1MB of flash and 256KB RAM on chip. It has a hardware floating point unit (FPU) and the configuration supports DSP instructions, single cycle multiply and accumulate hardware divide for energy efficient complex operations. The peripherals are designed to have a low power consumption ARM trust zone security. The nRF52840 also has firmware over the air upgrade facility.

In addition to sensors connected through GPIO, smart home sensors and controllers, Industrial IoT sensors, entertainment devices, gaming controllers, virtual and augmented reality applications can be connected through Bluetooth low energy capability of nRF52840.This in fact doubles the impact of processing using the nRF9160 DK kit.

Figure 9 shows the working configuration of the equipment where the kit connects to a cluster of bluetooth low energy devices through nRF52840. The Kit has GPS 
support through satellite. The kit collects data from a whole bunch of sensors and sends them to Google Cloud.

Analytics is now run on the events so generated and the necessary control commands initiated.

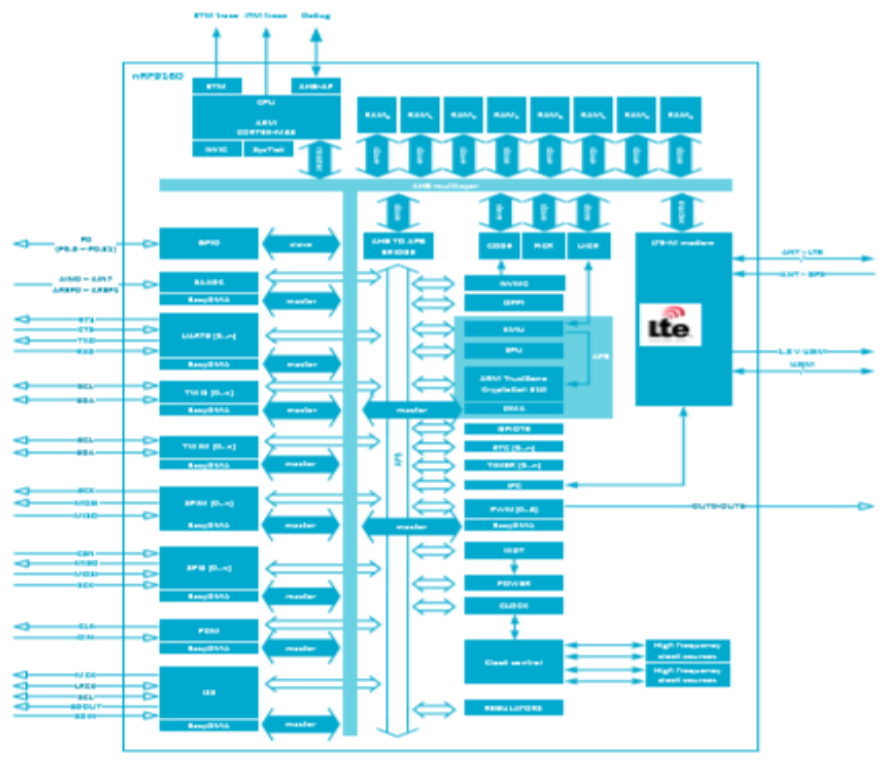

Fig. 8. Detailed architectural representation.

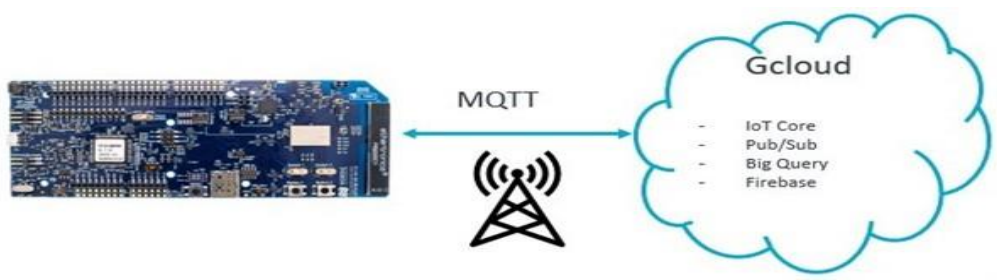

Fig. 9. MQTT interface with Cloud.

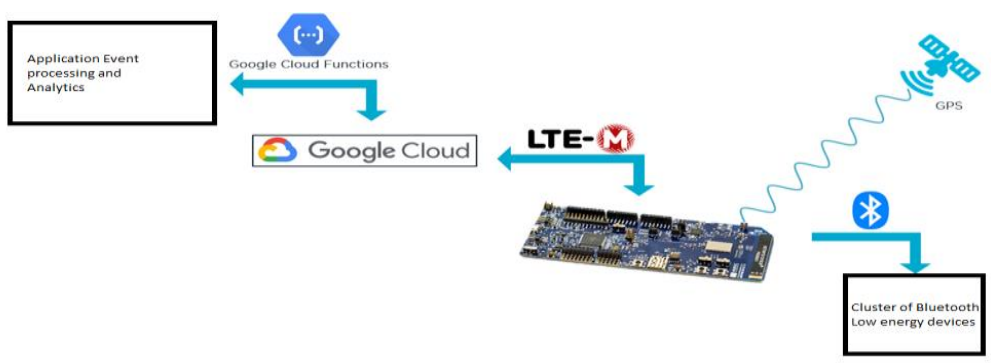

Fig. 10.Cloud based application development 


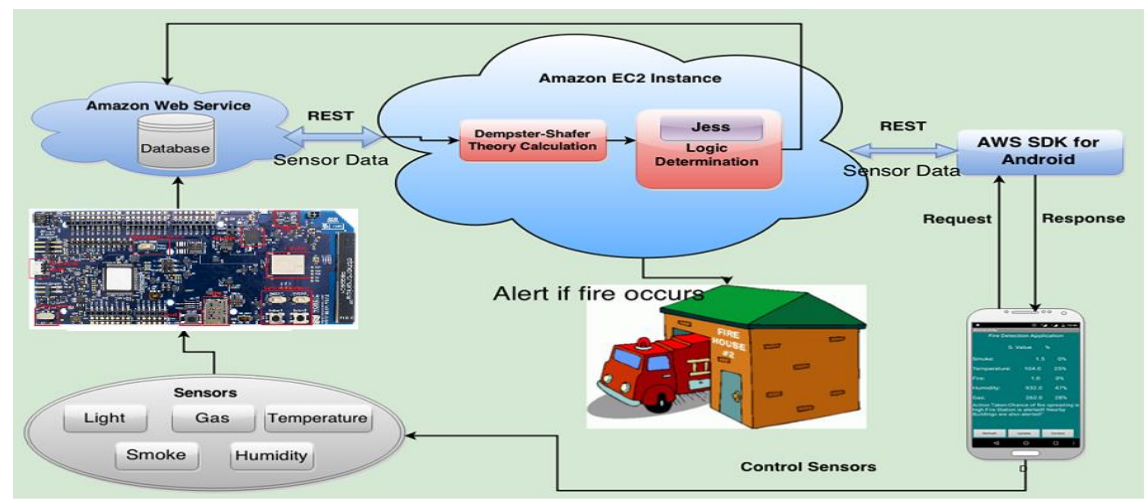

Fig. 11.A typical Smart Home use case

\section{Software Flow}

\subsection{Algorithm processing at kit}

- Install At command notification handler

- Subscribe network status notifications

- Configure system mode lte

- Example application started

- Print imei imsi

- The application is now connected

- Send a message to test connection

- Get binding to NRF cloud

- Initialise the Cloud application

- Cloud decode init is now performed.

- Work to be performed is initialised

- Modem is now configured

- Now a Cloud connect is made

- While (forever) \{

- Cloud keepalive time left

- $\}$

- At the end of the application a Cloud disconnect is made

\section{$4 \quad$ Results and Discussion}

As the range of cellular coverage is very large, let us assume that this kit is deployed in every state in India and the sensors interfaced with the kit measure rainfall. The total data is now aggregated at a central node which is equipped with a Python server used to perform data analytics. Here big data analytics can be used to decipher the data and an annual prediction for the coming years can be made 


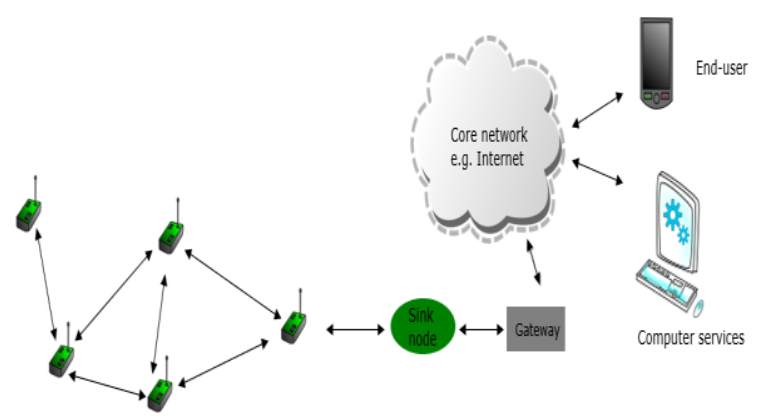

Fig. 12.A network of kits connected to a Gateway

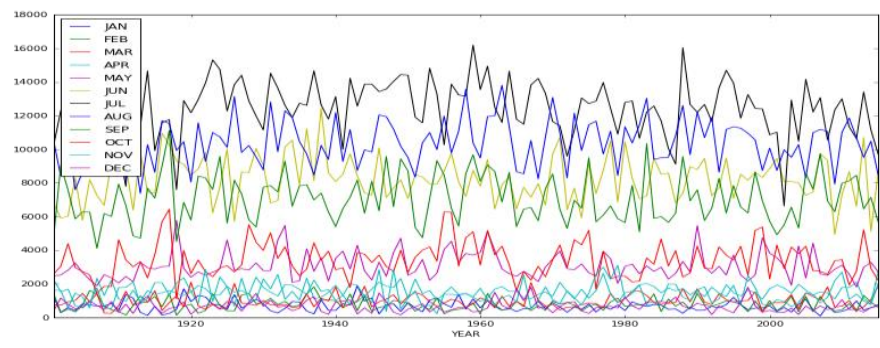

Fig. 13. Rainfall data for different months in the year

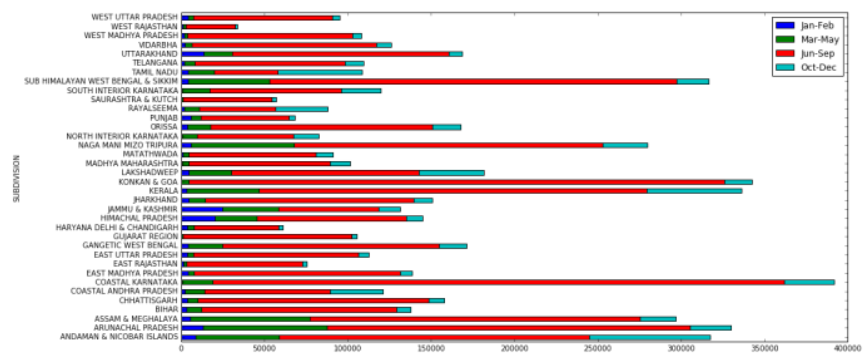

Fig. 14.State wise tabulation of Rainfall data

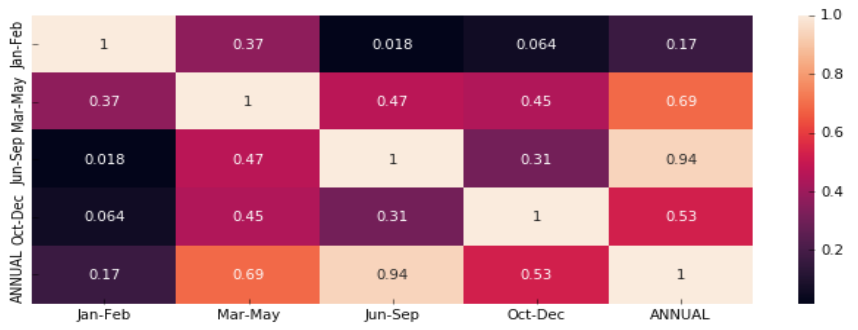

Fig. 15.Seasonal Heat map 


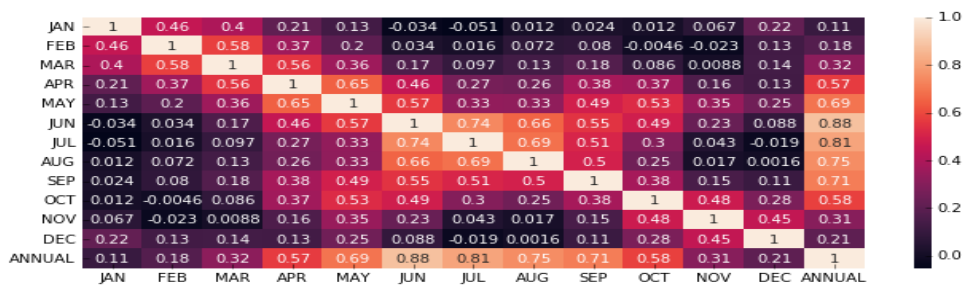

Fig. 16.Correlation (amounts of rainfall over months)

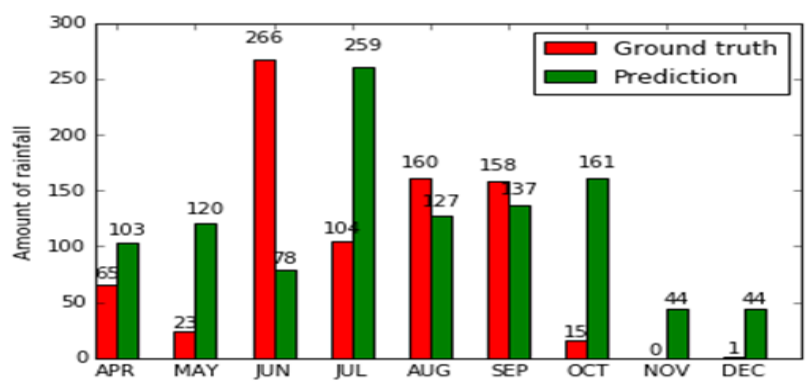

Fig. 17.Prediction for the coming years

Just like rainfall the air quality can also be monitored.

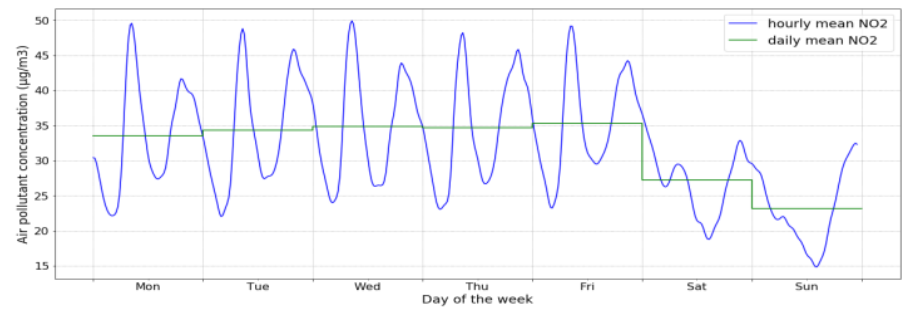

Fig. 18. Air Quality

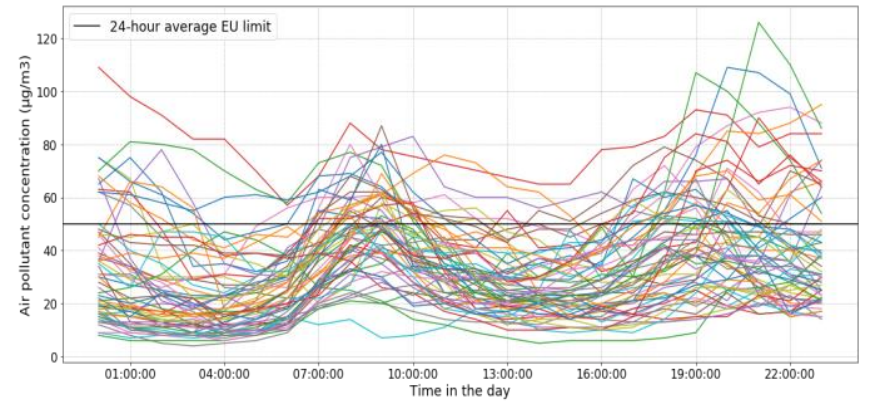

Fig. 19.Air Pollutant concentration 
In the same way the crime in every state can be monitored.
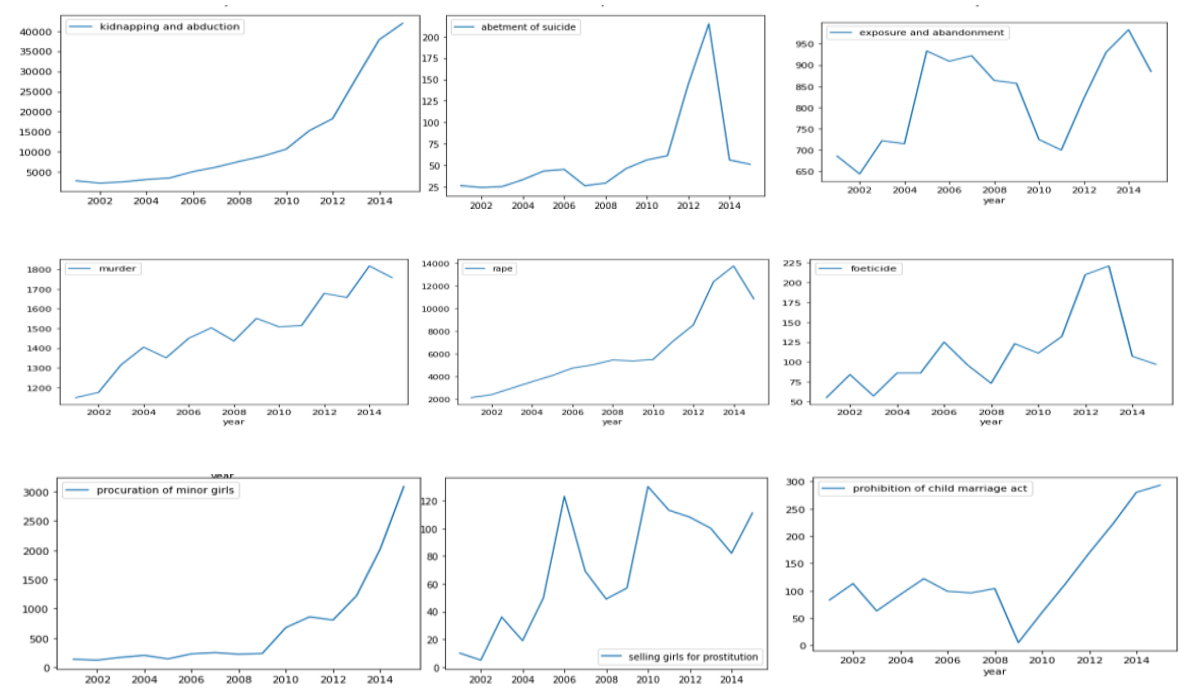

Fig. 20.Crime data (Overall)

The kit when deployed properly can facilitate smart city applications.

The service provider can determine the cellular coverage for the IoT service depending on what data rates the communication has to be supported, the device density, the need for battery for extended time periods, location of devices, devices with lots of fading, the mobility of the device nodes.

The choice of a network provider with coverage and connectivity at a fair price is required. As the devices are deployed over long periods of time there should be flexibility in selecting the network provider.

\section{Conclusion}

A low power wireless area network is implemented namely Cellular-IoT. This provides a robust long-range connection with short range wireless sensors. It provides a novel approach for a competing solution.

The nRF connect SDK helps you jump start your application. It makes use of Zephyr RTOS and communicates with CoAP, MQTT and LWM2M. This is an affordable single board development kit for nRF9160SP.It supports both SIM and ESIM.

The kit has been extensively tested with leading customers. It is a complete solution that integrates all the needs of a cellular connection and IOT networks. This will enable innovators to design products which can catalyze the development of smart city applications. 


\section{References}

[1] Benjamin Finley, Alexandr Vesselkov "Cellular IoT Traffic Characterization and Evolution" 2019 IEEE 5th World Forum on Internet of Things (WF-IoT) 978-1-5386-4980-0/19/\$31.00 (C)2019 https://doi.org/10.1109/wf-iot.2019.8767323

[2] Badr Eddine Benhiba, Abdessalam Ait Madi, Adnane Addaim"Comparative Study of The Various new Cellular IoT Technologies" 978-1-5386-4225-2/18/\$31.00 @2018 IEEE

[3] Sung Ho Chae, Sang-Woon Jeon, Cheol Jeong "Efficient Resource Allocation for IoT Cellular Networks in the Presence of Inter-band Interference": https://doi.org/10.1109/ tcomm.2019.2906230

[4] Taehoon Kim, Seong Ho Chae, "A Channel Estimator via Non-Orthogonal Pilot Signals for Uplink Cellular IoT" Digital Object Identifier https://doi.org/10.1109/access.2019.2912446

[5] Jubin Sebastian E, Axel Sikora, Manuel Schappacher, Zubair Amjad "Test and Measurement of LPWAN and Cellular IoT Networks in a Unified Testbed " 978-1-7281-2927-3 @2019 IEEE https://doi.org/10.1109/indin41052.2019.8972256

[6] Moussa, Weihua Zhuang "Energy and Delay Aware Two-hop NOMA-Enabled Massive Cellular IoT Communications" IEEE Internet of Things journal https://doi.org/10.1109/jiot.2019.2951584

[7] Sreekanth Dama, Thomas Valerrian Pasca, Vanlin Sathya, and Kiran Kuchi, "A Feasible Cellular Internet of Things" Digital Object Identifier 10.1109/MCE.2016.2614421 Date of publication: 14 December 2016

[8] Ali "O. Ercan, M. O־guz Sunay, Ian F. Akyildiz " RF Energy Harvesting and Transfer for Spectrum Sharing Cellular IoT Communications in 5G Systems", IEEE TRANSACTIONS ON MOBILE COMPUTING https://doi.org/10.1109/tmc.2017.2740378

[9] Mohammad Gharbieh, Hesham ElSawy, Ahmed Bader, Mohamed-Slim Alouini, "Spatiotemporal Stochastic Modeling of IoT Enabled Cellular Networks: Scalability and Stability Analysis ": IEEE https://doi.org/10.1109/tcomm.2017.2700309

[10] Hyunchul Joe, Hongsun An, Woowan Wang, Wonseok Lee, and Hyuncheol Park "In-band Cellular IoT for Smart Home Applications " 2017 IEEE International Conference on Consumer Electronics (ICCE) https://doi.org/10.1109/icce.2017.7889344

[11] Mustafa A. Kishk, Harpreet S. Dhillon "Joint Uplink and Downlink Coverage Analysis of Cellular-based RF-powered IoT Network", IEEE https://doi.org/10.1109/tgcn.2017. $\underline{2786694}$

\section{Author}

Arvind Vishnubhatla is a faculty member of Gokaraju Rangaraju institute of engineering and Technology Hyderabad. He is a $\mathrm{PhD}$ from Jawaharlal Nehru Technological University Hyderabad. He has vast experience in embedded systems and Dsp. Email: vainfo66@gmail.com

Article submitted 2020-10-01. Resubmitted 2020-10-28. Final acceptance 2020-10-28. Final version published as submitted by the authors. 